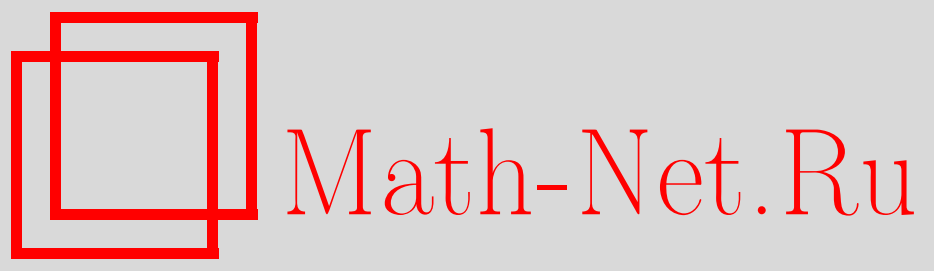

В. С. Кирчанов, Применение энтропии Реньи для описания квантовых диссипативных систем в статистической механике, ТМФ, 2008, том 156, номер 3, 444-453

DOI: https://doi.org/10.4213/tmf6258

Использование Общероссийского математического портала Math-Net.Ru подразумевает, что вы прочитали и согласны с пользовательским соглашением http://www . mathnet.ru/rus/agreement

Параметры загрузки:

IP : 54.172 .240 .79

26 апреля 2023 г., 13:09:23

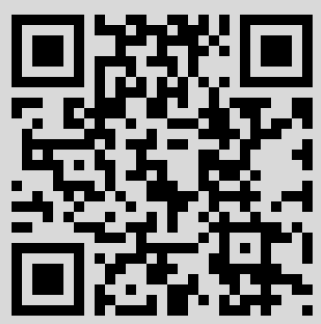




\title{
ПРИМЕНЕНИЕ ЭНТРОПИИ РЕНЬИ ДЛЯ ОПИСАНИЯ КВАНТОВЫХ ДИССИПАТИВНЫХ СИСТЕМ В СТАТИСТИЧЕСКОЙ МЕХАНИКЕ
}

\begin{abstract}
Развит формализм для описания квантовых диссипативных систем в статистической механике, опирающийся на квантовую энтропию Реньи. Из принципа максимума квантовой энтропии Реньи получено квантовое распределение Реньи. Дифференцированием квантового распределения Реньи (температурной матрицы плотности) по обратной температуре получено уравнение Блоха. Далее методом фейнмановского интеграла по траекториям с модифицированным функционалом Менского получено уравнение типа Линблада. С помощью проекционных операторов из этого уравнения выведено интегродифференциальное уравнение для сокращенного температурного статистического оператора, которое является аналогом уравнения Цванцига в статистической механике, и найдено его формальное решение в виде ряда в классе суммируемых функций.
\end{abstract}

Ключевые слова: квантовая энтропия Реньи, квантовое распределение Реньи, уравнение Блоха для квантового распределения Реньи, уравнение Линблада, уравнение Цванцига, квантовая диссипативная система.

\section{1. ВВЕДЕНИЕ}

Роль и значение энтропии в термодинамике, статистической физике и теории информации при описании сложных систем (например фрактальных и самоорганизующихся) превышает роль и значение энергии. Было предложено множество видов энтропии, отличающихся от классической энтропии Гиббса-Шеннона, в частности энтропия Реньи и $q$-энтропия (см. ссылки в работе [1]). В работах Башкирова, Витязева, Суханова [1]-[3] показано преимущество использования энтропии Реньи при описании сложных систем, так как она содержит энтропию Хаврда-Чарвата-Дароши-Тсаллиса (ХЧДТ) [4], [5] в качестве своей линеаризованной формы. Эволюция $q$-энтропии и диссипация энергии при необратимых процессах в неэкстенсивных системах рассмотрена в [6]. Ситуация в квантовой статистической механике несколько другая. При описании сложных квантовых систем здесь преобладает динамический временной подход, основанный на различных обобщениях квантового

*Пермский государственный технический университет, Пермь, Россия.

E-mail: kirchanovvs@pstu.ru 
уравнения Лиувилля (уравнения фон Неймана). Это связано с построением последовательной квантовой механики во фрактальных пространствах [7] и теорией квантовых измерений [8]. Следует также отметить важную работу Холево [9] о квантовых теоремах кодирования, где квантовая энтропия рассматривается в связи с прохождением информации по квантовому каналу связи. Возникает вопрос: как далеко можно продвинуться при описании квантовых диссипативных систем, если в качестве исходного пункта использовать квантовую энтропию Реньи?

Статья построена следующим образом. В разделе 2 из принципа максимума энтропии Джейнса выводится квантовое распределение Реньи. В разделе 3, дифференцируя квантовое распределение Реньи, получаем уравнение Блоха, которое в разделе 4 применяем для получения аналога уравнения Линблада для статистической механики. Мы используем идею Фейнмана, заключающуюся в том, что метод интегралов по траекториям в статистической механике оказывается проще, чем в квантовой механике (не появляются двойные коммутаторы). В разделе 5 мы получаем обобщенное уравнение Цванцига без мнимой единицы, используя для этого вновь выведенное уравнение типа Линблада. Во всех случаях динамической переменной является обратная температура.

\section{2. КВАНТОВОЕ РАСПРЕДЕЛЕНИЕ РЕНЬИ}

Энтропия Реньи без учета постоянной Больцмана $k_{\mathrm{B}}$ для квантовых систем имеет вид

$$
S_{\mathrm{R}}(\rho)=\frac{1}{1-q} \ln \operatorname{Tr} \rho^{q},
$$

где $\rho$ - статистический оператор (матрица плотности), $0<q \leqslant 1$ - параметр Реньи, условие нормировки

$$
\operatorname{Tr} \rho=1 .
$$

Квантовая энтропия Реньи переходит в обычную информационную энтропию при $q \rightarrow 1$

$$
\lim _{q \rightarrow 1} S_{\mathrm{R}}(\rho)=S_{\mathrm{inf}}[\rho]=-\operatorname{Tr}(\rho \ln \rho) .
$$

В случае выполнения условий работы [1] имеем

$$
\left|\operatorname{Tr} \rho^{q}-1\right| \ll 1, \quad|q-1| \ll 1 .
$$

Оставляя линейный член разложения логарифма в ряд в формуле (1), получаем квантовую энтропию ХЧДТ как линеаризованную форму квантовой энтропии Реньи

$$
S_{\mathrm{T}}(\rho)=\frac{1}{1-q}\left(\operatorname{Tr} \rho^{q}-1\right) .
$$

Тогда оператор квантовой микроскопической энтропии ХЧДТ принимает вид

$$
S_{\mathrm{T}}(\rho)=\frac{1-\rho^{1-q}}{1-q} .
$$


В случае $q=2$ квантовая энтропия Реньи при линеаризации логарифма превращается по терминологии Ло [10] в “информационную неопределенность" Брукнера-Зайлингера

$$
\left.S_{\mathrm{R}}\right|_{q=2}(\rho)=-\ln \operatorname{Tr} \rho^{2}=1-\operatorname{Tr} \rho^{2}, \quad 0<\operatorname{Tr} \rho^{2} \leqslant 2 .
$$

Энтропия ХЧДТ при $q=2$ также приводит к (3). Таким образом, энтропия Реньи включает в себя две практически важных линеаризированных формы.

Среднее значение гамильтониана $\widehat{H}$ имеет следующий вид:

$$
\langle\widehat{H}\rangle=\operatorname{Tr}(\widehat{H} \rho)=U .
$$

Для получения квантового распределения Реньи найдем абсолютный экстремум функционала $[11$, с. 58$]$

$$
L_{\mathrm{R}}[\rho]=\frac{1}{1-q} \ln \operatorname{Tr} \rho^{q}-\beta \operatorname{Tr}(\widehat{H} \rho)-\alpha \operatorname{Tr} \rho .
$$

Вариация функционала $\delta L_{\mathrm{R}}[\rho]=L_{\mathrm{R}}[\rho+\delta \rho]-L_{\mathrm{R}}[\rho]$ с учетом формулы $\delta \ln \operatorname{Tr} \rho^{q}=$ $q\left(\operatorname{Tr} \rho^{q}\right)^{-1} \operatorname{Tr}\left(\rho^{q-1} \delta \rho\right)$ принимает вид

$$
\delta L_{\mathrm{R}}[\rho]=\frac{q}{(1-q) \operatorname{Tr} \rho^{q}} \operatorname{Tr}\left(\rho^{q-1} \delta \rho\right)-\beta \operatorname{Tr}(\widehat{H} \delta \rho)-\alpha \operatorname{Tr} \delta \rho .
$$

Полагая $\delta L_{\mathrm{R}}[\rho]=0$, для произвольного $\delta \rho$ получаем равенство

$$
\frac{q}{(1-q) \operatorname{Tr} \rho^{q}} \rho^{q-1}-\beta U-\alpha=0 .
$$

Умножаем (5) на $\rho$ и применяем операцию $\mathrm{Tr}$. Тогда выражение для множителя Лагранжа принимает вид

$$
\alpha=\frac{q}{1-q}-\beta U
$$

Подставляя $\alpha$ в (5), получаем

$$
\frac{\rho^{q-1}}{\operatorname{Tr} \rho^{q}}-1-\frac{1}{q}(1-q) \beta \Delta \widehat{H}=0,
$$

где $\Delta \widehat{H}=\widehat{H}-U$. Из (6) следует, что

$$
\rho=\left(1-\frac{1}{q}(q-1) \beta \Delta \widehat{H}\right)^{1 /(q-1)}\left(\operatorname{Tr} \rho^{q}\right)^{1 /(q-1)} .
$$

Используя условие (2), окончательно получаем распределение Реньи для квантового случая

$$
\rho_{\mathrm{R}}(q, \beta)=\frac{1}{Z_{\mathrm{R}}}\left(1-\frac{1}{q}(q-1) \beta \Delta \widehat{H}\right)^{1 /(q-1)},
$$

где статистическая сумма квантового распределения Реньи имеет вид

$$
Z_{\mathrm{R}}(q, \beta)=\operatorname{Tr}\left(1-\frac{1}{q}(q-1) \beta \Delta \widehat{H}\right)^{1 /(q-1)} .
$$


Формулы (7) и (8) являются квантовым аналогом распределения Реньи, выведенного в работе [1] для классической энтропии Реньи.

При $q=2$ из (8) получаем квантовое распределение Брукнера-Зайлингера

$$
\rho_{\mathrm{R}}(2, \beta)=\frac{1-\beta \Delta H / 2}{\operatorname{Tr}(1-\beta \Delta H / 2)} .
$$

Если квантовое распределение Реньи (8) разложить в ряд, вычислить предел при $q \rightarrow 1$, применяя правило Лопиталя к каждому члену ряда, то получим эквивалентное квантовое каноническое распределение Гиббса

$$
\lim _{q \rightarrow 1} \rho_{\mathrm{R}}=\rho_{\mathrm{G}}=\frac{e^{-\beta \Delta \widehat{H}}}{\operatorname{Tr} e^{-\beta \Delta \widehat{H}}} .
$$

\section{3. УРАВНЕНИЕ БЛОХА ДЛЯ КВАНТОВОГО РАСПРЕДЕЛЕНИЯ РЕНЬИ}

Продифференцируем ненормированное квантовое распределение Реньи (8) по свободному параметру $\beta=1 / k_{\mathrm{B}} T$ :

$$
\frac{\partial \rho_{\mathrm{R}}}{\partial \beta}=\frac{\partial}{\partial \beta}(1-\beta A)=-(1-\beta A)^{\gamma-1} \gamma A=-\gamma \rho \rho^{-1 / \gamma} A,
$$

где $\gamma=1 /(q-1), A=(1 / q)(q-1) \Delta \widehat{H}$. В результате получим уравнение Блоха для квантового распределения Реньи

$$
q \frac{\partial \rho_{\mathrm{R}}(\beta)}{\partial \beta}=-\Delta \widehat{H} \rho_{\mathrm{R}}^{2-q}(\beta)
$$

с начальным условием в энергетическом представлении

$$
\left.\rho_{\mathrm{R}}(\beta)\right|_{\beta=0}=1 \text {. }
$$

В предельном случае при $q \rightarrow 1$ уравнение (10) переходит в эквивалентное уравнение Блоха для квантового канонического распределения Гиббса

$$
\lim _{q \rightarrow 1} \frac{\partial \rho_{\mathrm{R}}(\beta)}{\partial \beta}=\frac{\partial \rho_{\mathrm{G}}(\beta)}{\partial \beta}=-\Delta \widehat{H} \rho_{\mathrm{G}}(\beta)
$$

\section{4. УРАВНЕНИЕ ТИПА ЛИНБЛАДА ДЛЯ КВАНТОВЫХ СИСТЕМ С ЛИНЕЙНОЙ ДИССИПАЦИЕЙ}

Уравнение для квантового распределения Реньи в координатном представлении принимает вид

$$
\hbar(1-\eta) \frac{\partial \rho_{\mathrm{R}}\left(q, q^{\prime}, \nu\right)}{\partial \nu}=-\Delta \widehat{H} \rho_{\mathrm{R}}^{1+\eta}\left(q, q^{\prime}, \nu\right)
$$

где $\nu=\hbar / \beta=\hbar / k_{\mathrm{B}} T$ имеет размерность времени, $\eta=1-q-$ параметр порядка ${ }^{1)}$.

\footnotetext{
1) Мы переходим к параметру порядка $\eta$, чтобы далее использовать обозначения для обобщенных координат $q$ и импульсов $p$.
} 
Начальные условия для уравнения (10) следующие:

$$
\left.\rho_{\mathrm{R}}\left(q, q^{\prime}, \nu\right)\right|_{\nu=0}=\rho_{\mathrm{R} 0}\left(q, q^{\prime}\right) .
$$

Если $\widehat{H}=\widehat{H}(q(\nu), p(\nu))$, формальное решение уравнения (11), т.е. температурная матрица плотности (квантовое распределение Реньи), для отдельной траектории имеет вид

$$
\rho_{\mathrm{R}}\left(q, q^{\prime}, \nu\right)=\left[\left(\rho_{\mathrm{R} 0}\left(q, q^{\prime}\right)\right)^{-\eta}+\frac{\eta}{\hbar(1-\eta)} \int_{0}^{\nu} d \nu^{\prime}\left(\widehat{H}\left(p\left(\nu^{\prime}\right), q\left(\nu^{\prime}\right)\right)-U\right)\right]^{-1 / \eta} .
$$

Согласно теории квантовых измерений полная температурная матрица плотности может быть представлена многомерным фейнмановским интегралом по траекториям в форме ограниченного интеграла Менского [8]:

$$
\rho_{\mathrm{R}}\left(q, q^{\prime}, \nu\right)=\int d[a] \rho_{[a]},
$$

где

$$
\rho_{[a]}\left(q, q^{\prime}, \nu\right)=\int \cdots \int D q D p \rho_{\mathrm{R}}\left(q, q^{\prime}, \nu\right) w_{[a]}(p, q) .
$$

Модифицированный весовой функционал возьмем в виде

$$
\begin{aligned}
w_{[a]}(p, q)=\exp & \left\{-\int_{0}^{\nu} d \nu^{\prime}\left[\kappa\left(\widehat{A}(p, q)-a\left(\nu^{\prime}\right)\right)^{2}+\kappa a^{2}\left(\nu^{\prime}\right)+\right.\right. \\
+ & \left.\left.i \hbar^{-1} \lambda a\left(\nu^{\prime}\right) \widehat{B}(p, q)+2^{-1} \hbar^{-1} \widehat{C}(p, q)\right]\right\}
\end{aligned}
$$

где $\widehat{A}, \widehat{B}, \widehat{C}$ - операторы, $a\left(\nu^{\prime}\right)$ - результат измерения $\widehat{A}$ в момент $\nu^{\prime}, \kappa, \lambda$ - коэффициенты, $i$ - мнимая единица. Продифференцируем (12) по свободному параметру $\nu$ :

$$
\begin{aligned}
\frac{\partial \rho\left(q, q^{\prime}, \nu\right)}{\partial \nu}=\int & \ldots \int D p D q \frac{\partial \rho_{\mathrm{R}}}{\partial \nu} \int d[a] w_{[a]}(p, q)+ \\
& +\int \cdots \int D p D q \rho_{\mathrm{R}} \frac{\partial}{\partial \nu} \int d[a] w_{[a]}(p, q) .
\end{aligned}
$$

Используя формулу Лейбница дифференцирования интеграла по параметру и формулу (П.1), получаем

$$
\int d[a] w_{[a]}(p, q)=\sqrt{\frac{\pi}{\kappa}} \exp \left\{-\int_{0}^{\nu} d \nu^{\prime}\left[\widehat{A}^{2}+\frac{\lambda^{2}}{8 \kappa \hbar^{2}} \widehat{B}^{2}-\frac{i \lambda}{2 \hbar}(\widehat{A} \widehat{B}+\widehat{B} \widehat{A})+\frac{\widehat{C}}{2 h}\right]\right\} .
$$

Пренебрегая множителем $\sqrt{\pi / \kappa}$, получаем искомое уравнение типа уравнения Линблада в виде

$$
\frac{\partial \rho}{\partial \nu}=-\left(\widehat{\Omega}+\hbar^{-1} \widehat{C}\right) \rho-\frac{1}{\hbar(1-\eta)}(\widehat{H}-U) \int \cdots \int D p D q \rho_{\mathrm{R}}^{1+\eta} \int d[a] w_{[a]}(p, q),
$$

где $\widehat{\Omega}$ - квадратичный супероператор Линблада по диссипативным эрмитовым операторам $\widehat{A}$ и $\widehat{B}$ :

$$
\widehat{\Omega}=\frac{\kappa}{2} \widehat{A}^{2}+\frac{\lambda^{2}}{8 \kappa \hbar^{2}} \widehat{B}^{2}-i \frac{\lambda}{4 \hbar}(\widehat{A} \widehat{B}+\widehat{B} \widehat{A}) .
$$


Диссипативные операторы Линблада [12] $\widehat{L}, \widehat{L}^{+}$всегда можно представить через эрмитовы операторы $\widehat{A}$ и $\widehat{B}: \widehat{L}=\widehat{A}-i \widehat{B}, \widehat{L}^{+}=\widehat{A}+i \widehat{B}$. Квадратичный супероператор (14) проще аналогичного квадратичного супероператора в динамическом уравнении Линблада, так как он не содержит двойных коммутаторов от эрмитовых операторов $\widehat{A}$ и $\widehat{B}[13]$.

Чтобы получить замкнутое уравнение относительно $\rho$, положим $\rho_{\mathrm{R}}^{1+\eta}=\rho_{\mathrm{R}}+\Delta \rho_{\mathrm{R}}$, тогда уравнение (13) принимает вид

$$
\frac{\partial \rho}{\partial \nu}=-\frac{1}{\hbar(1-\eta)}(\widehat{H}-U)(\rho+\Delta \rho)-\left(\widehat{\Omega}+h^{-1} \widehat{C}\right) \rho,
$$

где

$$
\Delta \rho=\int \cdots \int D p D q \Delta \rho_{\mathrm{R}} \int d[a] w_{[a]}(p, q)
$$

Если представить статистический оператор $\rho_{\mathrm{R}}^{1+\eta}$ в виде ряда по параметру $\eta, 0 \leqslant$ $\eta<1$

$$
\rho_{\mathrm{R}}^{1+\eta}=\rho_{\mathrm{R}}\left[1-\left(1-\rho_{\mathrm{R}}\right)\right]^{1+\eta}=\rho_{\mathrm{R}}\left[1-\left(1-\rho_{\mathrm{R}}\right) \eta+\left(1-\rho_{\mathrm{R}}\right)^{2} \frac{\eta(\eta-1)}{2 !}-\cdots\right],
$$

то при $\eta=0, q=1$ и в линейном приближении $\Delta \rho_{\mathrm{R}}=\eta \rho_{\mathrm{R}}$ получаем уравнение (15) в виде

$$
\frac{\partial \rho}{\partial \nu}=-\hbar^{-1}(\Delta \widehat{H}+\widehat{C}) \rho-\widehat{\Omega} \rho
$$

Уравнение (17) не зависит от параметра порядка $\eta$ и эквивалентно уравнению Линблада для статистической механики [14], выведенному ранее из канонического распределения Гиббса. Таким образом, это уравнение остается справедливым даже в линейном приближении по параметру порядка.

\section{5. КВАНТОВОЕ УПРАВЛЯЮЩЕЕ УРАВНЕНИЕ ДЛЯ СОКРАЩЕННОГО СТАТИСТИЧЕСКОГО ОПЕРАТОРА}

Квантовое управляющее уравнение получим из уравнения (15), которое, не теряя общности, возьмем с оператором $\widehat{C}=0$ :

$$
\frac{\partial \rho}{\partial \nu}=-\widehat{F} \rho-\widehat{G} \Delta \rho
$$

где

$$
\widehat{F}=\widehat{G}+\widehat{\Omega}, \quad \widehat{G}=\frac{1}{\hbar(1-\eta)} \Delta \widehat{H} .
$$

Следуя подходу Цванцига [15], введем линейные проекционные операторы такие, что $P+Q=1, P Q=Q P$. Тогда сокращенные статистические операторы имеют вид

$$
\rho_{1}(\nu)=P \rho, \quad \rho_{2}(\nu)=Q \rho, \quad \rho_{1}+\rho_{2}=\rho .
$$

Выберем следующие начальные условия:

$$
\left.\rho_{1}(\nu)\right|_{\nu=0}=\rho_{10},\left.\quad \rho_{2}(\nu)\right|_{\nu=0}=0 .
$$


Применим к уравнению (16) последовательно операторы $P$ и $Q$ и, используя равенство (18), получаем следующую систему:

$$
\begin{aligned}
& \frac{\partial \rho_{1}}{\partial \nu}+P \widehat{F} \rho_{1}=-P \widehat{F} \rho_{2}-P \widehat{G} \Delta \rho, \\
& \frac{\partial \rho_{2}}{\partial \nu}+Q \widehat{F} \rho_{2}=-Q \widehat{F} \rho_{1}-Q \widehat{G} \Delta \rho .
\end{aligned}
$$

Решая уравнение (22) относительно $\rho_{2}$ и используя (19), получаем

$$
\rho_{2}=-\int_{0}^{\nu} e^{-Q \widehat{F} \nu^{\prime}} Q \widehat{F} \rho_{1}\left(\nu-\nu^{\prime}\right) d \nu^{\prime}-\int_{0}^{\nu} e^{-Q \widehat{F} \nu^{\prime}} Q \widehat{G} \Delta \rho\left(\nu-\nu^{\prime}\right) d \nu^{\prime} .
$$

Подставляя $(23)$ в $(21)$ и разделяя соотношения для $\rho_{1}$ и $\Delta \rho$, приходим к системе

$$
\begin{aligned}
\frac{\partial \rho_{1}}{\partial \nu}+P \widehat{F} \rho_{1}-P \widehat{F} \int_{0}^{\nu} e^{-Q \widehat{F} \nu^{\prime}} Q \widehat{F} \rho_{1}\left(\nu-\nu^{\prime}\right) d \nu^{\prime} & =-P \lambda\left(q, q^{\prime}\right), \\
P \widehat{F} \int_{0}^{\nu} e^{-Q \widehat{F} \nu^{\prime}} Q \widehat{G} \Delta \rho\left(\nu-\nu^{\prime}\right) d \nu^{\prime}-P \widehat{G} \Delta \rho & =-P \lambda\left(q, q^{\prime}\right),
\end{aligned}
$$

где $\lambda$ - параметр, не зависящий от $\nu$.

При $\lambda=0$ уравнение (25) является однородным интегральным уравнением Вольтерра второго рода и имеет единственное тривиальное решение $\Delta \rho(\nu) \equiv 0$ в классе суммируемых функций, а уравнение (24) становится интегродифференциальным уравнением типа уравнения Цванцига без мнимой единицы.

При $\lambda=\lambda\left(q, q^{\prime}\right)$ уравнение (25) имеет нетривиальное решение. Покажем это. Запишем уравнение (25) в виде

$$
Q \widehat{G} \Delta \rho(\nu)-Q \widehat{F} \int_{0}^{\nu} e^{-Q \widehat{F} \nu^{\prime}} Q \widehat{G} \Delta \rho\left(\nu-\nu^{\prime}\right) d \nu^{\prime}=Q \lambda
$$

с начальным условием

$$
\left.\Delta \rho\left(q, q^{\prime} \nu\right)\right|_{\nu=0}=\Delta \rho_{0}\left(q, q^{\prime}\right) .
$$

Обозначим $\varphi(\nu)=Q \widehat{G} \Delta \rho(\nu)$ и ядро уравнения $(26)$

$$
K\left(\nu^{\prime}\right)=\frac{d}{d \nu^{\prime}} e^{-Q \widehat{F} \nu^{\prime}}=-Q \widehat{F} e^{-Q \widehat{F} \nu^{\prime}} .
$$

Тогда получаем интегральное уравнение со сверткой

$$
\varphi(\nu)+K(\nu) * \varphi(\nu)=Q \lambda,
$$

где свертка

$$
K(\nu) * \varphi(\nu)=\int_{0}^{\nu} K\left(\nu^{\prime}\right) \varphi\left(\nu-\nu^{\prime}\right) d \nu^{\prime} .
$$

Применяя прямое преобразование Лапласа к (26),

$$
\begin{gathered}
\varphi(\nu) \circ-\bullet \Phi(s), \quad K(\nu) * \varphi(\nu) \circ-\bullet K(s) \Phi(s), \\
K(s)=s \frac{1}{s+Q F}-1, \quad Q \lambda \circ-\bullet \frac{1}{s} Q \lambda,
\end{gathered}
$$


получаем для изображения формулу

$$
\Phi(s)=\frac{1}{s} Q \lambda+\frac{1}{s^{2}} Q \widehat{F} Q \lambda .
$$

Применяя обратное преобразование Лапласа, получаем для оригинала формулу

$$
\varphi(\nu)=(1+\nu Q \widehat{F}) Q \lambda
$$

Возвращаясь к исходным обозначениям и используя начальное условие в (26), получаем

$$
\widehat{G} \Delta \rho_{0}\left(q, q^{\prime}\right)=\lambda\left(q, q^{\prime}\right)
$$

Тогда выражение (27) принимает вид

$$
\widehat{G} \Delta \rho(q, q, \nu)=(1+\nu \widehat{F} Q) \widehat{G} \Delta \rho_{0}\left(q, q^{\prime}\right) .
$$

Решение (29) справедливо для уравнения (25), взятого без проектора $P$, в следующем виде:

$$
\widehat{G} \Delta \rho-\widehat{F} \int_{0}^{\nu} e^{-Q \widehat{F} \nu^{\prime}} Q \widehat{G} \Delta \rho\left(\nu-\nu^{\prime}\right) d \nu^{\prime}=\widehat{G} \Delta \rho_{0}\left(q, q^{\prime}\right) .
$$

Решим теперь уравнение (24) с начальными условиями (20). Применим преобразование Лапласа

$$
\rho_{1}(\nu) \circ-\bullet R(s), \quad \frac{\partial \rho_{1}}{\partial \nu} \circ-\bullet s R(s)-\rho_{10}
$$

Для изображения $R(s)$ получаем уравнение

$$
R(s)=\frac{1}{s\left(1+P \widehat{F} \frac{1}{s+Q \widehat{F}}\right)} \rho_{10}+\frac{1}{s\left(1+P \widehat{F} \frac{1}{s+Q \widehat{F}}\right)} P \lambda .
$$

Введем обозначения $a=Q \widehat{F}, b=P \widehat{F}, x(s)=b /(s+a)$ и представим изображение в виде ряда $\left(x^{2}<1\right)$

$R(s)=\frac{1}{s}(1+x)^{-1} \rho_{10}+\frac{1}{s^{2}}(1+x)^{-1} P \lambda=\frac{1}{s}\left(1-x+x^{2}-\cdots\right) \rho_{10}+\frac{1}{s^{2}}\left(1-x+x^{2}-\cdots\right) P \lambda$.

Используя формулы (П.2)-(П.5) обратного преобразования Лапласа, получаем для оригинала выражение в виде ряда Неймана [16]

$$
\begin{aligned}
\rho_{1}(\nu)=\{1 & \left.-b\left(1-e^{-a \nu}\right) a^{-1}+b e^{-a \nu} * b\left(1-e^{-a \nu}\right) a^{-1}-\cdots\right\} \rho_{10}- \\
& -\left\{\nu-b\left[\nu a^{-1}-\left(1-e^{-a \nu}\right) a^{-2}\right]+b e^{-a \nu} * b\left[\nu a^{-1}-\left(1-e^{-a \nu}\right) a^{-2}\right]-\cdots\right\} P \lambda .
\end{aligned}
$$

Согласно (28) $P \lambda=P \widehat{G} \Delta \rho_{0}\left(q, q^{\prime}\right)$, поэтому окончательно получаем решение уравнения (24) в следующем виде:

$$
\rho_{1}(\nu)=\tilde{\rho}_{1}(\nu)-\Delta \tilde{\rho}_{1}(\nu),
$$


где

$$
\begin{aligned}
& \tilde{\rho}_{1}(\nu) \equiv\left\{1-P \widehat{F}\left(1-e^{-Q \widehat{F} \nu}\right)+\right. \\
& \left.+\int_{0}^{\nu} P \widehat{F} e^{-Q \widehat{F} \nu^{\prime}}\left[1-e^{-Q \widehat{F}\left(\nu-\nu^{\prime}\right)}\right](Q \widehat{F})^{-1} d \nu^{\prime}-\cdots\right\} \rho_{10}, \\
& \Delta \tilde{\rho}_{1}(\nu) \equiv\left\{\nu-P \widehat{F}\left[\nu(Q \widehat{F})^{-1}-\left(1-e^{-Q \widehat{F} \nu}\right)(Q \widehat{F})^{-2}\right]+\right. \\
& +\int_{0}^{\nu} P \widehat{F} e^{-Q \widehat{F} \nu^{\prime}} P \widehat{F}\left[\left(\nu-\nu^{\prime}\right)(Q \widehat{F})^{-1}-\right. \\
& \left.\left.-\left(1-e^{-Q \widehat{F}\left(\nu-\nu^{\prime}\right)}\right)(Q \widehat{F})^{-2}\right] d \nu^{\prime}-\cdots\right\} P \widehat{G} \Delta \rho_{0} .
\end{aligned}
$$

Формула (31) есть решение квантового управляющего уравнения (24) при $\lambda=0$. Полное формальное решение (30) квантовых управляющих уравнений (24) и (25) содержит дополнительное степенное слагаемое (32), так как мы использовали квантовое распределение Реньи (8) для получения уравнения типа Линблада (13) с супероператором (14).

\section{6. ЗАКЛЮЧЕНИЕ}

Таким образом, в работе показано, что, используя квантовую энтропию Реньи и динамическую переменную в виде обратной температуры, можно получить квантовое распределение Реньи и обобщенное уравнение Блоха. Методом фейнмановского интеграла по траекториям с модифицированным функционалом Менского получено уравнение типа Линблада в статистической механике. Применением проекционных операторов к этому уравнению выведено интегродифференциальное уравнение для сокращенного температурного статистического оператора, которое является аналогом уравнения Цванцига в статистической механике, и найдено его формальное решение в виде ряда в классе суммируемых функций. В пределе $q \rightarrow 1$ все полученные уравнения переходят в свои исходные уравнения. Отметим также, что полученные результаты справедливы, если исходить из квантовой энтропии ХЧДТ как частного случая энтропии Реньи.

\section{ПРИЛОЖКНИЕ}

Справедливы соотношения

$$
\int_{-\infty}^{\infty} \exp \left\{a\left(x_{1}-x\right)^{2}+a x^{2}-c x\right\} d x=\sqrt{\frac{-\pi}{2 a}} \exp \left\{\frac{a^{2} x_{1}^{2}-a c x_{1}-c^{2} / 4}{2 a}\right\} .
$$

Имеют место формулы обратного преобразования Лапласа:

$$
\begin{gathered}
\frac{1}{s} \bullet-\circ 1, \quad \frac{1}{s+a} \bullet-\circ e^{-a \nu}, \quad \frac{b}{s(s+a)} \bullet-\circ b\left(e^{-a \nu}-1\right) a^{-1}, \\
b\left(\frac{1}{s+a}\right) b\left(\frac{1}{s+a}\right)\left(\frac{1}{s}\right) \bullet-\circ b\left(e^{-a \nu}\right) * b\left(1-e^{-a \nu}\right) a^{-1}=
\end{gathered}
$$




$$
\begin{gathered}
=\int_{0}^{\nu} b e^{-a \nu^{\prime}} b\left(1-e^{-a\left(\nu-\nu^{\prime}\right)}\right) a^{-1} d \nu^{\prime}, \\
\frac{1}{s^{2}} \bullet-\circ \nu, \quad b\left(\frac{1}{s^{2}(s+a)}\right) \bullet-\circ b\left[a^{-2}\left(e^{-a \nu}-1\right)+\nu a^{-1}\right], \\
b\left(\frac{1}{s+a}\right) b\left(\frac{1}{s+a}\right) b\left(\frac{1}{s^{2}}\right) \bullet-\circ b e^{-a \nu} * b\left[\nu a^{-1}-\left(1-e^{-a \nu}\right) a^{-2}\right]= \\
=\int_{0}^{\nu} b e^{-a \nu^{\prime}} b\left[\left(\nu-\nu^{\prime}\right) a^{-1}-\left(1-e^{-a\left(\nu-\nu^{\prime}\right)}\right) a^{-2}\right] .
\end{gathered}
$$

\section{Список литературы}

[1] А. Г. Башкиров, ТМФ, 149:2 (2006), 299-317.

[2] А.Г. Башкиров, А. Д. Суханов, ЖЭТФ, 122:3 (2002), 513-520.

[3] A. G. Bashkirov, A. V. Vityazev, Phys. A, 277:1-2 (2000), 136-145.

[4] J. Havrda, F. Charvát, Kybernetika, 3 (1967), 30-35.

[5] C. Tsallis, J. Statist. Phys., 52:1-2 (1988), 479-487.

[6] Р. Г. Зарипов, Изв. вузов. Физика, 49:2 (2006), 35-41.

[7] V.E. Tarasov, Chaos, 14:1 (2004), 123-127.

[8] М. Б. Менский, Квантовые измерения и декогерениия. Модели и феноменология, Физматлит, М., 2001.

[9] А. С. Холево, УМН, 53:6(324) (1998), 193-230.

[10] Ло Шунь Лун, ТМФ, 151:2 (2007), 302-310.

[11] Д. Н. Зубарев, В. Г. Морозов, Г. Рёпке, Статистическая механика неравновесных процессов, Физматлит, М., 2002.

[12] G. Lindblad, Comm. Math. Phys., 48:2 (1976), 119-130.

[13] В. С. Кирчанов, ТМФ, 148:2 (2006), 288-294.

[14] В. С. Кирчанов, Изв. вузов. Физика, 49:12 (2006), 30-34.

[15] R. L. Zwanzig, J. Chem. Phys., 33 (1960), 1388-1341.

[16] Г. Дёч, Руководство $\kappa$ практическому применению преобразования Лапласа и Z-преобразования, Наука, М., 1971.

Поступила в редакцию 4.09.2007, после доработки 19.10.2007 